\title{
Mandibular metastasis of adenocarcinoma from prostate cancer: case report according to epidemiology and current therapeutical trends of the advanced prostate cancer
}

\author{
Juliana Dreyer da Silva de MENEZES ${ }^{1}$, Pietro Franchon Marques CAPPELLARI ${ }^{2}$, Marcos Maurício CAPELARI ${ }^{3}$, Paulo \\ Zupelari GONÇALVES ${ }^{4}$, Gustavo Lopes TOLEDO ${ }^{5}$, João Lopes TOLEDO FILHO ${ }^{6}$, Arsenio SALES-PERES ${ }^{7}$, Clóvis \\ MARZOLA ${ }^{8}$
}

\author{
1- Hospital for Rehabilitation of Craniofacial Anomalies, Bauru, SP, Brazil \\ 2- Santa Casa de Misericórdia Hospital, Ribeirão Preto, SP, Brazil. \\ 3- Department of Community Health, School of Oral and Maxillofacial Traumatology, Regional APCD, Bauru, SP, Brazil. \\ 4- Bauru Base Hospital Association, Bauru, SP, Brazil. \\ 5- Department of Biological Sciences, Bauru School of Dentistry, University of São Paulo; School of Oral and Maxillofacial Traumatology, Regional APCD, \\ Bauru, SP, Brazil. \\ 6- Department of Biological Sciences, Bauru School of Dentistry, University of São Paulo; School of Oral and Maxillofacial Traumatology, Regional APCD, \\ Bauru Base Hospital Association, Bauru, SP, Brazil. \\ 7- Department of Pediatric Dentistry, Orthodontics and Community Health, Bauru School of Dentistry, University of São Paulo, Bauru, SP, Brazil. \\ 8- Bauru School of Dentistry, University of São Paulo; School of Oral and Maxillofacial Traumatology, Regional APCD, Bauru, SP, Brazil.
}

Corresponding address: Juliana Dreyer da Silva de Menezes - Rua Aviador Gomes Ribeiro. 12-47/302 - Vila Noemy - Bauru - SP - Brasil - 17014-000 Phone: 551481265110 / 551431043544 - e-mail: dreyer.ju@gmail.com

Submitted: February 5, 2013 - Modification: May 24, 2013 - Accepted: June 4, 2013

\section{ABSTRACT}

\begin{abstract}
Drostate cancer represents the most frequent non-cutaneous neoplasia in males. This type of neoplasia can develop peculiar patterns of evolution, presenting, in many cases, precocious relapses and metastasis. Bone metastasis in the mouth is extremely rare, and represents $1 \%$ of all malignant mouth neoplasias. The aim of the present study is to report a clinical case of bone metastasis in the mandibular region associated with a tumoral prostate adenocarcinoma, as well as to discuss connected aspects about diagnosis, prognosis and integrated treatment of this condition.
\end{abstract}

Keywords: Mandible. Adenocarcinoma. Prostate cancer.

\section{INTRODUCTION}

Prostate cancer represents the most frequent non-cutaneous neoplasia in males, corresponding to $40 \%$ of the cases, being the second death cause due to malignant tumors in men. The incidence as much as the mortality increases exponentially after 50 years of age $5,9,10$.

This type of neoplasia can develop peculiar patterns of evolution, presenting, in many cases, precocious relapse and metastasis. Of a total of patients who have received curative treatment for the localized prostate cancer, $30 \%$ relapse. The most frequent is the bone metastasis, generally multiple, mainly reaching the vertebral column, shoulder joints and sacroiliac bone. Among these patients, survival generally varies from 20 to 30 months ${ }^{5,10}$.

Bone metastasis in the mouth is extremely rare, and represents $1 \%$ of all malignant mouth neoplasias. The incidence is 80 to $90 \%$ in the mandible, mainly in the molar region and it always sign the spreading of the cancer illness. It originates from primary malignant neoplasias located mainly in the breast, prostate, lung, thyroid and kidney. However, the occurrence of prostate and kidneys as primary fields for jaw's metastasis through blood pathways are considered extremely rare ${ }^{1,16}$. Among the bone metastases affecting the oral cavity, adenocarcinoma is the most frequent histological type ${ }^{1,16,23}$.

The main clinical manifestations may include 
pain, swelling, tooth loss, bleeding, trismus, paresthesia sensation (often associated with inferior alveolar nerve involvement) and epistaxis ${ }^{19}$. Included in the differential diagnosis of metastatic peripheral lesions on the gingiva or alveolar ridge are pyogenic granuloma, peripheral giant cell granuloma and, possibly, peripheral ossifying fibroma or ulcerated fibroma ${ }^{6}$. The difficulty of clinical identification and differentiation between primary lesions and metastatic lesions of the jaw make the biopsy with histopathological analysis (combined with immunohistochemistry) mandatory for diagnosis ${ }^{1}$.

Besides representing great importance for public health, this issue becomes fundamental for a multidisciplinary evaluation of the patient.

\section{CASE REPORT}

Male patient, white, 54 years old, was admitted in the Oral and Maxillofacial Trauma and Surgery Team at Hospital de Base de Bauru (Bauru Base Hospital), in March 2009. The patient's main complaint was pain in the left mandibular region above the median line and paresthesia of the anatomical areas sensitized by the Left Alveolar Inferior Nerve, branch of the Mandibular Nerve and Trigeminal Nerve. The first signal related by the patient was a pathology originated from a root treatment about one year before, which developed a mandibular abscess clinically treated. Once healed from this abscess, chronic pain persisted in half mandible associated with localized paresthesia. Because of the posterior absence of dental symptoms even as mandibular pain and face paresthesia, the clinical dentist referred the patient to the one who suggested the diagnosis of Trigeminal Neuralgia, establishing treatment with Carbamazepin 200 mg and Gabapentin 300 mg, both once a day, that lasted about 1 year or more.

The patient's medical history revealed pharmacologically controlled diabetes and prostate acinar adenocarcinoma Gleason score $7(3+4)$, with initial PSA score on 4.78. This latter treated in 2005 by means of radical prostatectomy performed in January 2006 (pT3NOM0), having follow-up procedures with urology, but in a random and intermittent manner, a history not explored or valued by neurology.

Physical examination revealed left mandibular bulging in the buccolingual aspect and tooth mobility involving bulging region extending to the opposite canine tooth associated with a slight gums erythema, but without symptoms compatible with periodontal disease. In addition, we observed the absence of pathognomonic signs and symptoms of classic Trigeminal Neuralgia, showing no intermittent pain, neither specific localized pain spots (trigger-points). Based on this clinical symptomatology, we established a hypothesis of diagnosis as osteomyelitis and/or cancer, not yet discarding the possibility of Atypical Neuralgia of the Trigeminal Nerve (Figure 1).

Through a radiography exam, it was possible to define a mild, diffuse radiolucency in the left mandibular body. The Computed Tomography Scan showed an anatomical structure alteration with osteolysis areas in the premolars, molars and in the left region of the chin (Figures 2 and 3 ).

Because of that, we carried out an incisional biopsy and culture/antibiogram of sensitivity of the affected area. The results showed adenocarcinoma (clear cell type) with necrosis areas and infiltrating bone and soft tissue. The isolated microorganism was Staphylococcus aureus. These findings suggest

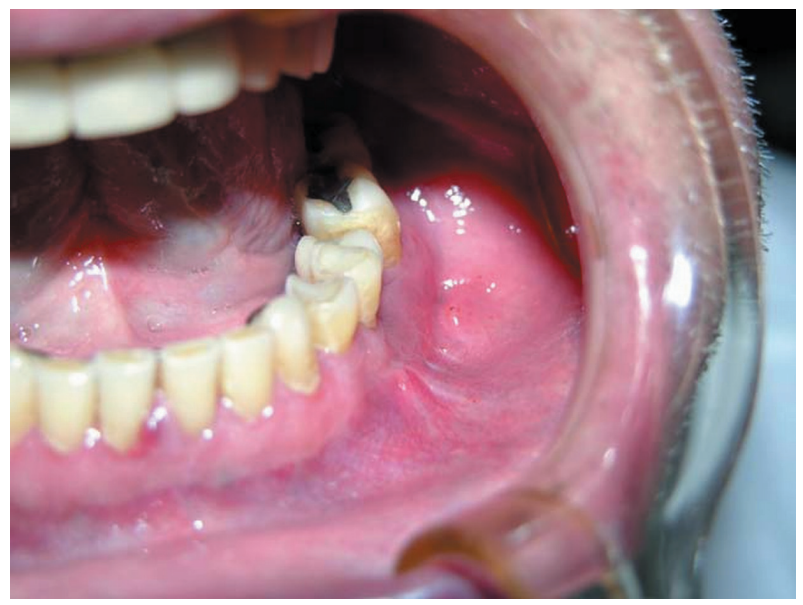

Figure 1- Clinical aspect of the patient. It is possible to see the bulging involving left buccolingual region of premolars and molars. Source: Oral and Maxillofacial Surgery team - Bauru Base Hospital Association

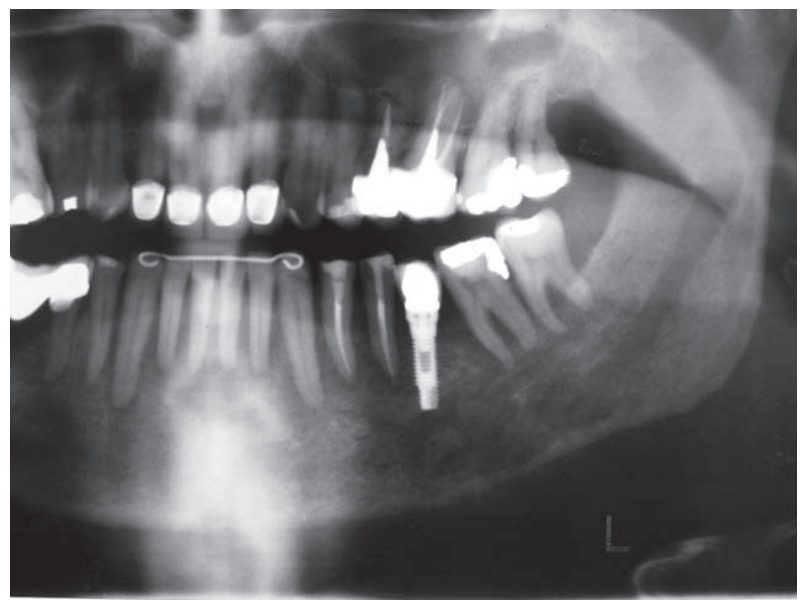

Figure 2- Panoramic radiography showing diffuse radiolucent area in the left mandibular body region. Source: Oral and Maxillofacial Surgery team - Bauru Base Hospital Association 
a metastatic tumor infected by via hematogenous route. The tumor had no communication between the internal and external environment, without skin or mucosa fistula, confirmed by whole-body bone scintigraphy with special interest in the mandibular region, where increased uptake of the radiocontrast agent was observed, as well as in many areas, such as the right clavicle and scapula, humerus, ribs, spine, sternum, hip bone and femur (right/ left) (Figure 4).

After confirming a definitive diagnosis, the patient was taken to the Oncology Clinic, where in May 2009

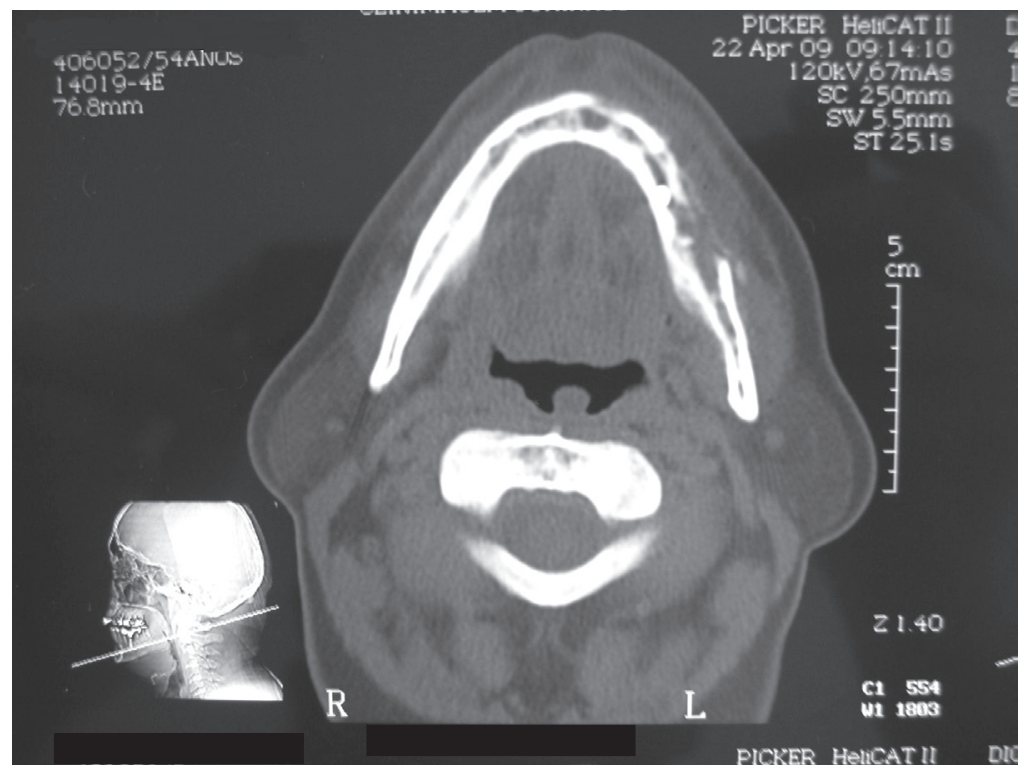

Figure 3- Computed tomography showing areas of osteolysis in the left mandibular body and symphysis region. Source: Oral and Maxillofacial Surgery team - Bauru Base Hospital Association

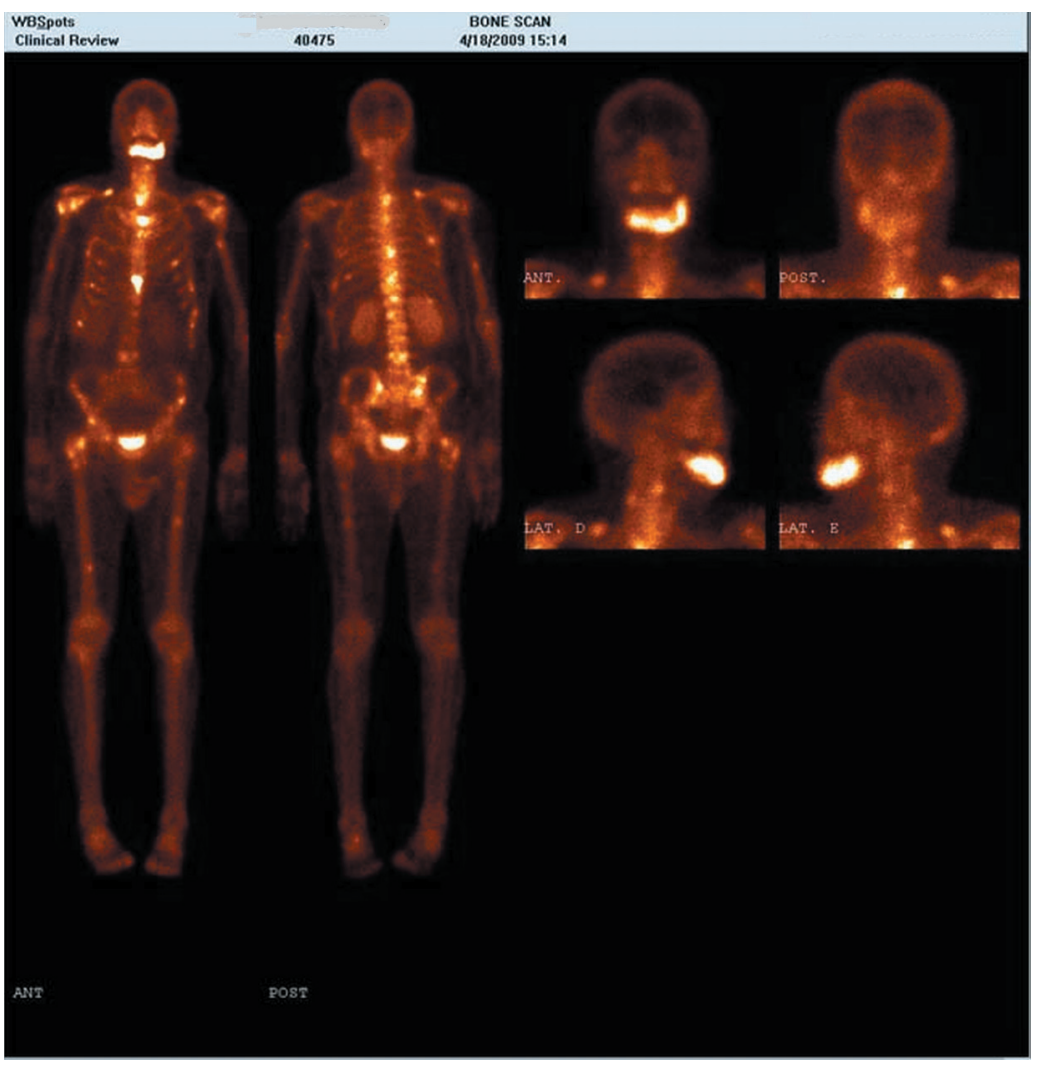

Figure 4- Whole-body bone scintigraphy, increased uptake of the radiocontrast agent observed in the mandibular region, right clavicle and scapula, humerus, ribs, spine, sternum, hip bone and femur (right/left). Source: Oral and Maxillofacial Surgery team - Bauru Base Hospital Association 
was admitted to the Clinical Oncology Department with a metastatic bone disease diagnosis (prostate acinar adenocarcinoma) confirmed by three different methods: bone scintigraphy, incisional biopsy and immunohistochemical analysis of mandibular lesions. The treatment consisted of the use of goserelin acetate $(3.6 \mathrm{mg} / \mathrm{month})$ and bisphosphonate (clodronate, 4 ampoules/month). The patient was evaluated as having good disease control and good tolerance to treatment for some months, when disseminated neoplasm and pulmonary involvement led to a lethal outcome, with the patient's death in less than a year.

\section{DISCUSSION}

Prostate cancer is a silent disease; the findings show that the prevalence of histological malignancy exceeds the clinically manifest disease in approximately eight times. According to the American Cancer Society, for early cancer detection in individuals without symptoms, it is recommended the digital rectal exam and PSA dosage annually for individuals over age 50 or 45 for men belonging to risk groups ${ }^{18}$.

The PSA is a kallikrein family protease, produced by the prostate epithelium, whose function is to solubilize the sperm after ejaculation. The accepted level as the upper limit of normal PSA is $4 \mathrm{ng} / \mathrm{ml}^{18}$. The PSA has a great clinical utility; it serves for early detection of prostate cancer, cancer staging, prognostic assessment and therapeutical response control ${ }^{3}$.

In addition to the PSA level in case of established disease, it is necessary to evaluate the prostate cancer advancement by image exams [transrectal ultrasound, bone scintigraphy, chest radiography, computed tomography of the abdomen and pelvis, magnetic nuclear resonance of the abdomen and pelvis, and positron emission tomography (PET)] to define the appropriate therapeutical strategy. In order to classify neoplastic levels, some methods are used to analyze the histological grade (Gleason score), tumor volume and stage at the diagnosis time ${ }^{12-14,16}$.

When the primary tumor volume is less than $3 \mathrm{ml}$, metastasis rarely occurs. When the present volume is greater than $12 \mathrm{ml}$ metastasis is almost always present ${ }^{5,16}$. According to McNeal ${ }^{11}$ (1992), only tumors larger than $1 \mathrm{~cm}$ in diameter (volume of $0.5 \mathrm{ml}$ ) are capable of extracapsular extension. The lymph nodes or seminal vesicles spread are usually more common in tumors larger than $2 \mathrm{~cm}$ in diameter ${ }^{11}$.

The Gleason system has been introduced to aid on the tumor evolution prediction and the pathological staging by transrectal biopsy or of the surgical specimen itself. The lesions with
Gleason score 2-4 (well differentiated) have a more indolent behavior, whereas the ones scoring 7-10 (undifferentiated) are aggressive, responding poorly to various treatment options ${ }^{6}$.

The clinical staging of tumors, TNM classification, has been adopted to characterize tumors, helping in choosing the most appropriate therapy and to estimating the patients' survival chances. This system evaluates the primary tumor, regional lymph nodes and distant metastase ${ }^{19}$.

The patients' selection for specific treatments is usually done through the risk of tumor recurrence and dissemination subdivision. Among the therapeutical modalities there are clinical observation, surgery, radiotherapy, chemotherapy, hormonal therapy and combination therapy.

Rutsatz, et al. ${ }^{17}$ (1990) in a period of 35 years, reported 1008 patients with craniofacial malignancies, found only five patients $(0.5 \%)$ with metastatic tumors to the jaw, from different primary sites. Van der Waal, et al. ${ }^{23}$ (2003), identified adenocarcinoma as the primary histological type responsible for gnathic bone metastases, starting from different locations of primary tumors, confirming the reported case.

The four most common tumor sites that metastasize to the man jaws, in descending order of frequency, are lung, prostate, kidney and liver and, in women, breast, adrenal gland, female genital organs (uterus, cervix, ovaries) and colorectum. In the first decade of life, tumors of the adrenal gland (neuroblastoma) most frequently spread to the jaws and in the second decade, bone primaries prevail (osteosarcoma, Ewing's/PNET) ${ }^{8}$. Most patients with oral metastasis, generally have the primary cancer well diagnosed, confirming the reported case in which the primary tumor was treated years before by radical prostatectomy and the patient was still on medical care during the gnathic metastasis. In $25-35 \%$ of cases, the presence of metastasis on the head and neck is the first sign of disseminated malignant disease, and in about $70 \%$ of cases, metastatic lesions are diagnosed in conjunction with the primary focus. Sometimes, however, the diagnosis of the primary lesion may be difficult or even impossible 2,21.

Multidisciplinary work is recommended in the care of patients with malignant neoplasms or neurological disorder of doubtful identification in order to provide diagnosis, planning and treatment that offer the most favorable prognosis.

Piattelli, et al. ${ }^{15}(1999)$ conducted a survey of 390 oral cancer cases, of which 22 cases (5.6\%) were metastatic tumors on gnathic bones from other regions, being prostate gland the primary site of these tumors, as occurred in our case.

In cases of metastatic bone illness, the pain and pathological fractures are prevalent manifestations, 
although the condition is asymptomatic in some patients. In this case, the patient reported the presence of chronic pain in hemi mandible, including a localized paresthesia, symptoms that were initially treated as Trigeminal Neuralgia. However, by conducting special examinations, we obtained the diagnosis of metastatic adenocarcinoma prostate cancer in the lung and bone, involving, in addition to the cited organs, the mandibular region. Bone tumor involvement of the oral region is higher compared to the soft tissue. Metastatic disease in the jaws may also affect the adjacent soft tissues and present as dental or periodontal infection ${ }^{20}$. When the metastasis is located in the jaw, the primary neoplasm is more likely to develop bone metastases, especially in the molar region ${ }^{1}$. It is believed that the greatest amount of cancellous bone present in the region is the reason for the higher frequency of involvement when compared to other facial bones, as trabecular bone presents growth factors that improve the neoplasia spread, confirming the reported case in which bone metastasis reached the region of the body and the mandibular symphysis ${ }^{8}$.

The unilateral paresthesia type, as occurred in this case, hypoesthesia or anesthesia of the lower lip, known as Numb Chin Syndrome with or without the presence of pain, may be the initial clinical manifestation of a significant percentage that manifests on the jaw. Alterations in sensitivity involving the lower lip, even without significant radiological changes, should alert the expert to think about the possibility of an initial metastasis ${ }^{1,21}$. For an accurate diagnosis and treatment of this rare condition, detailed and careful evaluation of the clinical picture, associated with a high degree of suspicion, is needed, requiring a multidisciplinary approach to the case ${ }^{8,21}$.

The prognosis of these patients is reserved for the pathophysiology of the primary tumor. Usually, with minimal cellular differentiation they may render the initial site location. In addition, bone metastases are associated with advanced disease and in some cases may be involved in malignant lesions - which may lead the patient to death, as reported in this case ${ }^{7}$.

The radiation treatment can be considered curative regarding single oral metastases whose primary focus is clearly identified and which responded well to therapy. However, most authors consider an unfavorable prognosis for most cases of this oral cancer ${ }^{2}$. Surgical treatment in most cases is not recommended, indicating palliative therapy. The cases that may have a better indication are those with localized metastases, with no other identified metastasis and controlled primary disease, which does not occur in our case, since the examination of bone scintigraphy identified multiple metastatic tumors distributed throughout the body, palliative therapy being considered the best alternative ${ }^{14}$.

Chemotherapy is indicated only in metastatic disease refractory to hormonal treatment, as in cases of predominance of hormone-independent cell clones or progressive increase in serum PSA ${ }^{22}$. The use of bisphosphonates, as reported in the present case, is justified in the presence of bone metastases and when refractory to hormonal treatment, despite the risk of complications related to these drugs, such as osteonecrosis. There is an interesting indication to the use of these drugs as this therapy provides control of the neoplastic disease and also a relative patient survival chance ${ }^{4}$. In advanced cases, the quality of life, despite the patients having no chance of cure for the disease, is the main objective, providing less pain and discomfort ${ }^{17}$.

\section{FINAL CONSIDERATIONS}

Metastatic lesions are related to the advanced stage of the disease and usually with a high degree of histological aggressiveness. This fact explains the difficulty of curative treatment, and these injuries are usually classified as reserved and dark prognosis. It is important to emphasize the relevance of detailed clinical examination and multidisciplinary evaluation in order to establish the differential diagnosis and so the correct treatment.

\section{REFERENCES}

1- Amonett MR, McGinnis JP Jr, Krolls SO. Metastasis to the mandible. Miss Dent Assoc J. 1998;54(2):36-9.

2- Antunes AA, Antunes AP. Gnathic bone metastasis: a retrospective study of 10 cases. Rev Bras Otorrinolaringol. 2008;74(4):561-5.

3- Bill-Axelson A, Holmberg L, Ruutu M, Garmo H, Stark JR, Busch $C$, et al. Radical prostatectomy versus watchful waiting in early prostate cancer. N Engl J Med. 2011;364(18):1708-17.

4- Catalona WJ, Antenor JAV, Roehl KA, Moul JW. Screening for prostate cancer in high risk populations. J Urol. 2002;186:1980-4. 5- Clarke BM, Boyette J, Vural E, Suen JY, Anaissie EJ, Stack BC Jr. Bisphosphonates and jaw osteonecrosis: the UAMS experience. Otolaryngol Head Neck Surg. 2007;136(3):396-400.

6- D'Silva NJ, Summerlin DJ, Cordell KG, Abdelsayed RA, Tomich $\mathrm{CE}$, Hanks $\mathrm{CT}$, et al. Metastatic tumors in the jaws: a retrospective study of 114 cases. J Am Dent Assoc. 2006;137:1667-72.

7- Harrison D, Lund VJ. Tumors of the upper jaw. New York: Churchil Livingstone; 1993.

8- Hirshberg A, Shnaiderman-Shapiro A, Kaplan I, Berger R. Metastatic tumours to the oral cavity - pathogenesis and analysis of 673 cases. Oral Oncol. 2008;44:743-52.

9- INCA - National Cancer Institute of Brazil [database]. Rio de Janeiro: Ministry of Health, Federal Government - Brasil; [cited 2009 Sep 01]. Available from: http://www.inca.gov.br/ conteudo_view.asp?id=339.

10- Jemal A, Thomas A, Murray T, Thun M. Cancer statistics, 2002. CA Cancer J Clin. 2002;52:23-47.

11- McNeal JE. Cancer volume and site of origin of adenocarcinoma of the prostate: relationship to local and distant spread. Hum Pathol. 1992;23:258-66. 
12- Memon AG, Jaleel A, Aftab J. Pattern of prostatic carcinoma metastases in bones detected by bone scans using Technitium 99m Methyl Diphosphate (TC99m MDP) imaging technique. Pak J Med Sci. 2006;22(2):180-3.

13- Narayan P, Gajendran V, Taylor SP, Tewari A, Presti JC Jr, Leidich $R$, et al. The role of transrectal ultrasound-guided biopsy-based staging, preoperative serum prostate-specific antigen, and biopsy Gleason score in prediction of final pathologic diagnosis in prostate cancer. Urology. 1995;46:205-12.

14- Partin AW, Kattan MW, Subong EN, Walsh PC, Wojno KJ Oesterling $\mathrm{JE}$, et al. Combination of prostate-specific antigen, clinical stage, and Gleason score to predict pathological stage of localized prostate cancer. A multi-institutional update. JAMA. 1997;277:1445-51.

15- Piattelli A, Fioroni M, Rubini C. Gingival metastasis from a prostate adenocarcinoma: report of a case. J Periodontol. $1999 ; 70(4): 441-4$.

16- Pruckmayer M, Glaser C, Marosi C, Leitha T. Mandibular pain as the leading clinical symptom for metastatic disease: nine cases and review of the literature. Ann Oncol. 1998;9(5):559-64.
17- Sandler HM, Eisenberger MA. Assessing and treating patients with increasing prostate specific antigen following radical prostatectomy. J Urol. 2007;178(3):S20-4.

18- Smith RA, Cokkinides V, Eyre HJ. American Cancer Society guidelines for the early detection of cancer, 2006. CA Cancer J Clin. 2006;56:11-25.

19- Sobin LH, Wittekind Ch, editors. TNM classification of malignant tumours. $6^{\text {th }}$ ed. New York: Wiley-Liss; 2002.

20- Stege R. Potential side-effects of endocrine treatment of long duration in prostate cancer. Prostate Suppl. 2000;10:38-42.

21- Tamiolakis D, Tsamis I, Thomaidis V, Lambropoulou M, Alexiadis G, Venizelos I, et al. Jaw bone metastases: four cases. Acta Dermatovenerol Alp Panonica Adriat. 2007;16 (1):21-5. 22- Tannock IF, de Wit R, Berry WR, Horti J, Pluzanska A, Chi KN, et al. Docetaxel plus prednisone or mitoxantrone plus prednisone for advanced prostate cancer. N Engl J Med. 2004;351(15):1502-12. 23- Van der Waal RI, Buter J, Van der Waal I. Oral metastases: report of 24 cases. Br J Oral Maxillofac Surg. 2003;4(1):3-6. 